\title{
The Uplink Capacity Evaluation of Wireless Networks: Spectral Analysis Approach
}

\author{
Abdellatif Kobbane ${ }^{1}$, Rachid El-Azouzi ${ }^{2}$, Khalil Ibrahimi ${ }^{2,3}$, \\ Sujit Kumar Samanta ${ }^{2}$ and El-Houssine Bouyakhf ${ }^{3}$ \\ ${ }^{1}$ ENSIAS, Mohammed V-Souissi University, Rabat, Morocco \\ 2 LIA/CERI, University of Avignon, France \\ 3 LIMIARF/FSR, Mohammed V-Agdal University, Rabat, Morocco
}

In this paper we study the capacity of wireless cellular network, in particular the uplink of WCDMA system by using the two dimensional continuous-time Markov chain (CTMC) technique. Considering two types of calls: real-time (RT) calls characterized by a quasi fixed transmission rate, and best-effort $(\mathrm{BE})$ calls which do not require strict demand but need some reliability conditions, we develop an approach based on the spectral analysis for evaluating the cell capacity. We explicitly obtain the simultaneous distribution of the number of RT connections and the number of BE connections in the steady-state. This analysis allows us to simplify the computation of the performance measures including expected delay and throughput of BE traffic. These performances are obtained explicitly in both cases (finite and infinite) of $\mathrm{BE}$ calls as function of system parameters like arrival rate of $\mathrm{BE}$ and $\mathrm{RT}$ calls, service rate of BE and RT calls. These results allow the operator to evaluate the cell capacity by varying these parameters independently of the number of BE calls according to its policy to manage the network. We further propose some CAC (Call Admission Control) policies for BE traffic. We finally conclude this work with some numerical and simulation results. The simulation results obtained by the network simulator (NS2) are closely related to the numerical results of our analytical results which validate our theoretical model.

Keywords: spectral analysis approach, call admission control, wireless networks, Matrix-Geometric method

\section{Introduction}

The Universal Mobile Telecommunication System (UMTS) operates with Wideband Code Division Multiple Access (WCDMA) over the air interface. The advantage of the third generation
(3G) of mobile networks resides in the fact that they offer to users a large possibility of services. These services are related to real-time (RT) and best-effort (BE) applications like transferring files, emailing, etc. Each service has a demand of quality of service. The variations caused by the diversity of classes can affect the WCDMA capacity.

Many works have been developed in the field of capacity analysis for wireless networks. Several research axes on WCDMA capacity have been considered. Zhang and Yue [36] presented a method to calculate the WCDMA reserve link Erlang based on the Lost Call Held (LCH) models as described by Viterbi [33]. This algorithm calculates the occupancy and capacity of UMTS/WCDMA systems based on a system outage condition. In this paper, the authors derive a closed form expression of Erlang capacity for a single type of traffic. The capacity of an uplink with two classes is considered by Mandayam et al. [22] in which the real-time traffic is transmitted all the time and the non-real-time or the best-effort traffics are time-shared. Altman [2] considered best-effort and real-time traffic and studied the influence of the value of a fixed (non-adaptive) bandwidth per BE calls on the Erlang capacity of the system (that includes also RT calls), taking into account that a lower bandwidth implies longer call durations. Hegde and Altman [15] extended the notion of capacity in [2] to other quality of service (QoS). The delay aware capacity, in particular suitable for the 
$\mathrm{BE}$ traffic, is defined as the arrival rate of $\mathrm{BE}$ calls that the system can handle so that their expected delay is bounded. Admission control in third generation UMTS systems was the focus of the works done by Elayoubi et al. [12,13] for uplink, and by Elayoubi et al. [11] for downlink. They specifically calculated the system capacity for three different types of receivers, namely Matched Filter, Minimum Mean Square Error (MMSE) and decorrelator, and taking into account the classical signal-to-interference (SIR), coverage and mobility. They also implemented several call admission control (CAC) algorithms that handle priorities between handoff flows and new ones as well as voice calls versus data ones. Using two approaches: a proactive one based on measurement, and a reactive one based on squeezing of data flows so as to accommodate higher priority calls.

The capacity of the Orthogonal Frequency-Division Multiple Access (OFDMA) and CodeDivision Multiple Access (CDMA) ranging subsystems in 802.16 has been studied in a few papers. Seo et al. [32] analyzed the performance about random access protocol which uses ranging subsystem in OFDMA-CDMA environment with respect to mean delay time (MDT) and first exit time (FET). Cho et al. [17] designed and analyzed the performance analysis of the model to control adaptively the size of each ranging code for initial ranging (IR), periodic ranging $(\mathrm{PR})$ and bandwidth request (BR) ranging in order to efficiently do random access. You and Kim [35] evaluated the capacity of a ranging subsystem in terms of the ranging code error probability versus the number of active users to attempt ranging. Recently, several works addressing QoS in general and CAC have been produced. For instance, an admission control scheme is proposed by Wang et al. [34]. It ensures highest priority to unsolicited grant services (UGS) flows while maximizing overall bandwidth by means of bandwidth borrowing. Chen et al. [9] treated the QoS based on classical intserv and diffserv paradigms as well as their mapping to IEEE 802.16 MAC layer. All these works assumed a fixed user scenario.

In this paper, we consider an uplink WCDMA system with two types of calls: real-time (RT) calls that have dedicated resources, and besteffort (BE) traffic without any strict QoS requirement. Our analysis is based on the modeling of the system as a two dimensional Markov chain, where the first corresponds to the number of RT calls and the second to the number of BE calls. In order to obtain the steady-state distribution of the number of RT calls and BE calls of this system, we make use of advanced spectral analysis of quasi-birth-and-death (QBD) processes $[27,23,14,31,8,25,24]$. In particular, we obtain the steady-state probability of the Markov chain as a function of the eigenvalues and eigenvectors of some finite matrices. We obtain an explicit solution which has not been available in [15]. These results allow us to express some performance measures of interest as a function of eigenvalues and eigenvectors of some matrices. Since the RT calls need a minimum guaranteed transmission rate, the system can handle a finite number of RT calls. However, we show that these eigenvalues and eigenvectors are obtained independently of the number of BE calls which reduces the computation complexity. An important application of our approach is to reduce the computation complexity of CAC policy for BE calls. Hence, our approach is useful to service provider to obtain the CAC policy of BE traffic with low computation complexity that maximizes the bandwidth utilization and satisfies the QoS required by RT calls and BE calls.

The main contributions in this paper are to analyze the system capacity of real wireless networks like WCDMA, HSPA and WiMAX based on the spectral analysis approach, and also to find the closed form of interest metric performances (blocking probability and delay), independently of the number of BE calls as function only of the number of RT calls, and system parameters (arrival rates and service rates of both RT and BE calls). The spectral analysis approach allows us to find these results by using the eigenvalues and eigenvectors of finite rate matrices. These results allow the operator to evaluate the cell capacity by varying these parameters independently of the number of $\mathrm{BE}$ calls according to its policy to manage the network in which the complexity of CAC is reduced. Furthermore, the providing theoretical formulas by spectral analysis are validated with the network simulator NS2.

The rest of this paper is organized as follows: Section 2 presents the problem formulation. We analyze the single cell case of the system in Section 3. Section 4 shows a detailed spectral analysis of the system with both finite and infinite 
numbers of BE calls. In Section 5, we extend the analysis to the multi-cell case. Section 6 gives explicitly the performance measures of interests. Numerical results are given in Section 7 and support the results of the analysis. Finally, we perform extensive simulation results and verify that the analytical results closely match the results obtained from simulations.

\section{Problem Formulation}

Let $H=\{1, \ldots, C\}$ be the set of multi-service classes in an uplink of a WCDMA system with multi-sectors. Let $M_{i}$ be the number of mobiles of class $i$ in a sector, where $i=1,2, \ldots, C$. We refer to the received power from a mobile of class $i$ at the base station in a sector by $P_{i}$. This power is the same for all mobiles of class $i$ and the following signal to interference ratio (SIR) expression should be satisfied in order to have uplink communication $[15,20]$ :

$$
\frac{P_{i}}{N+I_{\text {own }}+I_{\text {other }}-P_{i}}=\varrho_{i} \geq\left(\frac{E_{i}}{N_{0}} \frac{R_{i}}{W}\right)=\chi_{i},
$$

where $i=1,2, \ldots, C ; N$ is the background noise density; $E_{i}$ is the energy per transmitted bit of type $i ; R_{i}$ is the transmission rate of class $i$ service; $W$ is the spread-spectrum bandwidth; $N_{0}$ is the terminal noise density; $I_{\text {own }}$ is the total power received from mobiles belonging to the same sector and $I_{\text {other }}$ denotes the total power received from mobiles in other sectors. By definition, the intra- and inter-cells interference in a sector are, respectively, given by

$$
\begin{aligned}
I_{\text {own }} & =\sum_{j=1}^{C} M_{j} P_{j}, \\
I_{\text {other }} & =g I_{\text {own }},
\end{aligned}
$$

where $g$ is the constant of interference given from measurement [20]. The equation (1) is valid if the power control in WCDMA is perfect. Due to inaccuracy in the closed-loop fast power control and for shadow fading of the radio channel, the $\varrho_{i}$ is not all times equal to $\left(\frac{E_{i}}{N_{0}} \frac{R_{i}}{W}\right)$. Then $\varrho_{i}$ is a random variable with lognormal distribution of the form $\varrho_{i}=10^{0.1 \xi_{i}}$, where $\xi_{i} \sim N\left(\mu_{\xi}, \sigma_{\xi}\right)$ includes the shadow fading component and $\sigma_{\xi}$ (resp. $\mu_{\xi}$ ) is the standard deviation (resp. mean) of shadow fading. In order to take into account the shadow fading effect, the authors in [15] introduced a new constant $\Gamma$ independent of the service class. Then the condition in (1), i.e., $\varrho_{i}$ can be modified instantaneously by $\left(\frac{E_{i}}{N_{0}} \frac{R_{i}}{W} \Gamma\right)$, where

$$
\begin{gathered}
\Gamma=10^{\frac{\sigma_{\xi}^{2}}{20 h}-\frac{\sigma_{\xi} Q^{-1}(\beta)}{10}}, \\
\beta=P\left(\varrho_{i}>\chi\right)=\int_{\chi_{i}}^{\infty} f_{\varrho_{i}}(x) d x=Q\left(\frac{h \ln \chi-\mu_{\xi}}{\sigma_{\xi}}\right), \\
f_{\varrho_{i}}(x)=\frac{h}{x \sigma_{\xi} \sqrt{2 \pi}} \exp \left(-\frac{h\left(\ln x-\mu_{\xi}\right)^{2}}{2 \sigma_{\xi}^{2}}\right), \\
h=\frac{10}{\ln 10}, Q(x)=\int_{x}^{\infty} \frac{e^{-t^{2} / 2}}{2 \pi} d t .
\end{gathered}
$$

So the signal to interference ratio must be larger than or equal to $\left(\frac{E_{i}}{N_{0}} \frac{R_{i}}{W} \Gamma\right)$. Then, for a better satisfaction of calls of class $i$ without degradation in QoS and in order to serve a large number of users, the minimal received power $\left(P_{i}\right)$ must satisfy the following equation [3]:

$$
P_{i}=\frac{N \Delta_{i}}{1-(1+g) \sum_{j=1}^{C} M_{j} \Delta_{j}}, i=1, \ldots, C,
$$

where $\Delta_{j}=\frac{E_{j} R_{j} \Gamma}{N_{0} W+E_{j} R_{j} \Gamma}$. The load rate is defined as follows

$$
\theta=\sum_{j=1}^{C} M_{j} \Delta_{j}
$$

We pick up the total load $\theta$ from (5) and put it in (4), we get the minimal power $\left(P_{i}\right)$ in terms of total load $(\theta)$ as

$$
P_{i}=\frac{N \Delta_{i}}{1-(1+g) \theta} .
$$

This power must be positive finite and hence it means that the denominator is strictly positive. We denote the system capacity by $\Theta_{\varepsilon}$ and it is defined as the upper bound of $\theta$ (i.e., $\left.\theta \leq \Theta_{\varepsilon} /(1+g)\right)$, where $\Theta_{\varepsilon}=1-\epsilon$ and $\epsilon$ is a very small positive number.

The value of $\Gamma$ is a function of the standard deviation $\left(\sigma_{\xi}\right)$ of the shadow fading of users, whose value varies with user mobility. The multi-paths fading due to only user mobility (user speed) are not considered in this paper. But our model is still valid in any case of shadowing, where only the value of $\Gamma$ may be changed. 


\section{Single Cell Case}

Here, we first restrict our work to two service classes in single sector so that there is no interference from other sectors, i.e., $g=0$.

We consider that UMTS uses the Adaptive MultiRate (AMR) [16] codec for RT calls. The minimum transmission rate of RT calls is denoted by $R_{R T}^{m}$ and the upper bound transmission rate is denoted by $R_{R T}^{r}$. The bandwidth $\Delta_{R T}^{k}$ corresponding to rate $R_{R T}^{k}$ is given by

$$
\Delta_{R T}^{k}=\frac{\Gamma E_{R T} / N_{0}}{W / R_{R T}^{k}+\Gamma E_{R T} / N_{0}}, k=r, m,
$$

where $E_{R T}$ is the energy per bit transmitted for $\mathrm{RT} ; N_{0}$ is the terminal noise density and $W$ is the WCDMA modulation bandwidth. The capacity available for $\mathrm{BE}$ calls depends on the current number of RT calls in the system. We model an uplink of WCDMA system by two-dimensional Markov chain processes $\left(X_{R T}(t), X_{B E}(t)\right)$, where $X_{R T}(t)$ (resp. $\left.X_{B E}(t)\right)$ represents the number of calls of the type RT (resp. BE) in the system at time $t$. Calls of class RT (resp. BE) arrive according to a Poisson process with density $\lambda_{R T}$ (resp. $\lambda_{B E}$ ) and their duration is exponentially distributed with parameter $\mu_{R T}$ (resp. $\mu_{B E}$ ).

The minimal portion reserved for the $\mathrm{BE}$ calls is denoted by $L_{B E}$. Let $M_{R T}$ be the maximum number of RT calls that can be served simultaneously by the system and it is given by $M_{R T}=\left|\frac{L_{R T} T}{\Delta_{R T}^{T}}\right|$, where $|x|$ means the largest integer part of $x$ and $L_{R T}$ denotes the remaining maximum capacity for RT calls and it is given by

$$
L_{R T}=\Theta_{\varepsilon}-L_{B E} .
$$

Hence, the capacity used by BE calls when there is $i_{R T}$ of RT calls in progress is $C\left(i_{R T}\right)$ and given by

$$
C\left(i_{R T}\right)=\left\{\begin{array}{l}
\Theta_{\varepsilon}-i_{R T} \Delta_{R T}^{r}, \quad \text { if } 1 \leq i_{R T} \leq N_{R T}, \\
L_{B E}, \text { if } N_{R T}<i_{R T} \leq M_{R T}
\end{array}\right.
$$

where $N_{R T}=\left|\frac{L_{R T}}{\Delta_{R T}}\right|$ is the number of RT calls corresponding to the normalized capacity required by RT call $\left(\Delta_{R T}^{r}\right)$. Note that the RT calls are independent on the $\mathrm{BE}$ calls. Thus, the RT calls can be modeled by $M / M / M_{R T} / M_{R T}$ queue with an arrival rate $\lambda_{R T}$ of RT calls and exponential call duration of mean value $1 / \mu_{R T}$. From well-known (e.g., [1]) balance equations of the underlying Markov chain of this queue, the stationary probabilities $q_{i}=\lim _{t \rightarrow \infty} \operatorname{Pr}\left[X_{R T}(t)=i\right]$ can be rewritten recursively as:

$$
q_{i+1}=\frac{\rho_{R T}}{i+1} q_{i}, i=0,1, \ldots, M_{R T},
$$

where $\rho_{R T}=\frac{\lambda_{R T}}{\mu_{R T}}$. Using equation (10) and the normalising condition $\sum_{i=0}^{M_{R T}} q_{i}=1$, we have

$$
q_{i}=\frac{\left(\rho_{R T}\right)^{i} / i !}{\sum_{j=0}^{M_{R T}} \frac{\left(\rho_{R T}\right)^{j}}{j !}}, i=0,1, \ldots, M_{R T} .
$$

Then the blocking probability of RT calls is given by Erlang formula [10] as

$$
P_{B}^{R T}=q_{M_{R T}}=\frac{\left(\rho_{R T}\right)^{M_{R T}} / M_{R T} !}{\sum_{j=0}^{M_{R T}} \frac{\left(\rho_{R T}\right)^{j}}{j !}} .
$$

If we consider both RT calls and BE calls, then the state of the system is defined by a Markov chain $\left(X_{R T}(t), X_{B E}(t)\right)$. Let $E^{(\infty)}$ denote the infinite space and is given by

$$
E^{(\infty)}=\left\{(i, j): 0 \leq i \leq M_{R T}, j \geq 0\right\},
$$

where the symbol $\infty$ indicates that the number of $\mathrm{BE}$ calls is infinite. The service time of $\mathrm{BE}$ call is

$$
v(i)=\mu_{B E} R_{B E}(i),
$$

where $R_{B E}(i)$ is the total rate of $\mathrm{BE}$ calls which corresponds to the use of the capacity $C(i)$ as follows:

$$
R_{B E}(i)=\frac{C(i)}{1-C(i)} \frac{W}{\Gamma E_{B E} / N_{0}},
$$

where $E_{B E}$ is the energy of $\mathrm{BE}$ calls. The Markov chain is ergodic if and only if the average service time of $\mathrm{BE}$ calls is greater than the BE load, i.e.,

$$
\mu_{B E} E\left[R_{B E}(i)\right]>\lambda_{B E},
$$

where $\mu_{B E} E\left[R_{B E}(i)\right]=\sum_{i=1}^{M_{R T}} v(i) q_{i}$. The infinitesimal generator of this system is described by a homogeneous quasi-birth-and-death process $\left(\mathrm{QBD}\right.$ ) denoted by $\mathbf{Q}^{(\infty)}$ (Latouche and 
Ramaswami[21]). It can be written as

$$
\mathbf{Q}^{(\infty)}=\left(\begin{array}{ccccc}
\mathbf{B} & \mathbf{A}_{0} & \mathbf{0} & \ldots & \ldots \\
\mathbf{A}_{2} & \mathbf{A}_{1} & \mathbf{A}_{0} & \ddots & \ldots \\
\mathbf{0} & \mathbf{A}_{2} & \mathbf{A}_{1} & \mathbf{A}_{0} & \ddots \\
\vdots & \ddots & \ddots & \ddots & \ddots \\
\vdots & \ddots & \ddots & \ddots & \ddots
\end{array}\right)
$$

where $\mathbf{A}_{0}, \mathbf{A}_{1}, \mathbf{A}_{2}$ and $\mathbf{B}$ are square matrices of order $M_{R T}+1$ and are given by

$$
\begin{aligned}
\mathbf{A}_{0}= & \operatorname{diag}\left(\lambda_{B E}\right), \\
\mathbf{A}_{2}= & \operatorname{diag}(v(i)) ; i=0,1,2, \ldots, M_{R T}, \\
\mathbf{A}_{1}[i, i+1]= & \lambda_{R T} ; i=0,1,2, \ldots, M_{R T}-1, \\
\mathbf{A}_{1}[i, i-1]= & i \mu_{R T} ; i=1,2, \ldots, M_{R T}, \\
\mathbf{A}_{1}[i, i]= & -\left(\lambda_{B E}+\lambda_{R T}+i \mu_{R T}+v(i)\right) \\
& i=0, \ldots, M_{R T}-1, \\
\mathbf{A}_{1}\left[M_{R T}, M_{R T}\right]= & -\left(\lambda_{B E}+M_{R T} \mu_{R T}+v\left(M_{R T}\right)\right), \\
\mathbf{B}= & \mathbf{A}_{1}+\mathbf{A}_{2} .
\end{aligned}
$$

In our study, we focus also on the quality of service required by BE calls. Let $M_{B E}$ be the maximum number of $B E$ calls that can be handled by the system for a given QoS required by BE calls. This QoS corresponds to a given expected delay of $\mathrm{BE}$ call so that it does not exceed a positive constant which defines a CAC for best effort calls. Hence, the system will be described by a homogeneous QBD (Latouche and Ramaswami [21]) Markov chain $\left(X_{R T}(t), X_{B E}(t)\right)$. Then, the finite space denoted by $E^{(f)}$ is

$$
E^{(f)}=\left\{(i, j): 0 \leq i \leq M_{R T}, 0 \leq j \leq M_{B E}\right\},
$$

where the symbol $f$ indicates that the number of the BE calls is finite. The infinitesimal generator $\mathbf{Q}^{(f)}$ of this system can be written as

$$
\mathbf{Q}^{(f)}=\left(\begin{array}{cccccc}
\mathbf{B} & \mathbf{A}_{0} & \mathbf{0} & \ldots & \ldots & \mathbf{0} \\
\mathbf{A}_{2} & \mathbf{A}_{1} & \mathbf{A}_{0} & \ddots & \ddots & \vdots \\
\mathbf{0} & \ddots & \ddots & \ddots & \ddots & \vdots \\
\vdots & \ddots & \ddots & \ddots & \ddots & \mathbf{0} \\
\vdots & \ldots & \ddots & \mathbf{A}_{2} & \mathbf{A}_{1} & \mathbf{A}_{0} \\
0 & \ldots & \ldots & \mathbf{0} & \mathbf{A}_{2} & \mathbf{F}
\end{array}\right)
$$

where $\mathbf{F}=\mathbf{A}_{1}+\mathbf{A}_{0}$, and $\mathbf{B}, \mathbf{A}_{1}, \mathbf{A}_{0}$ and $\mathbf{A}_{2}$ are same as mentioned above for the infinite case.

Remark 1 We note that our spectral analysis approach is still valid in other wireless networks such as WiMAX/HSPA in both downlink and uplink. In this case, we can find a new SIR and derive the transmission rate of BE calls. Of course, this rate will be different, which depends on other parameters like scheduling, number of available codes, adaptive modulation and coding scheme (AMC), inter- and intra-cell interference. For example, we can use our approach to analyze the system that joint uplink and downlink simultaneously in HSDPA, where the QBD form is given by Altman et al. [4] and also in WiMAX by Chahed et al. [7].

We recall that our aim in this work is to find a closed form of the steady-state probability of the Markov chain as a function of the eigenvalues and eigenvectors of some finite matrices. These forms are needed in order to express some performance measures of interest (blocking probability and sojourn time of BE calls) as a function of eigenvalues and eigenvectors of some matrices as explained in the next section.

\section{System Analysis}

In this section, we use the advanced method of spectral analysis of quasi-birth-and-death process (Latouche and Ramaswami [21]). We study in detail the usage of spectral analysis for computing the steady-state distribution in two cases when the number of BE calls is infinite as well as finite.

\section{The Case for Infinite Number of BE Calls}

Let $\pi_{i, j}^{(\infty)}$ be the steady-state probability of the Markov chain $\left(X_{R T}(t), X_{B E}(t)\right)$ defined as

$$
\pi_{i, j}^{(\infty)}=\lim _{t \rightarrow \infty} \operatorname{Pr}\left[X_{R T}(t)=i, X_{B E}(t)=j\right]
$$

Under the stability condition, the generator matrix $\mathbf{Q}^{(\infty)}$ is irreducible and aperiodic. Hence the steady-state distribution of this Markov chain is the unique solution of the following equations:

$$
\begin{gathered}
\boldsymbol{\Pi}^{(\infty)} \mathbf{Q}^{(\infty)}=\mathbf{0}, \\
\boldsymbol{\Pi}^{(\infty)} \mathbf{e}^{(\infty)}=1,
\end{gathered}
$$

where $\mathbf{e}^{(\infty)}$ is a column vector of the ones with an appropriate dimension and $\boldsymbol{\Pi}^{(\infty)}$ is the block 
of steady-state probability vector in the following form:

$$
\boldsymbol{\Pi}^{(\infty)}=\left(\boldsymbol{\Pi}_{0}^{(\infty)}, \boldsymbol{\Pi}_{1}^{(\infty)}, \ldots, \boldsymbol{\Pi}_{j}^{(\infty)}, \ldots\right)
$$

where $\Pi_{j}^{(\infty)}=\left(\pi_{0, j}^{(\infty)}, \ldots, \pi_{M_{R T}, j}^{(\infty)}\right)$. The equation (19) can be rewritten as:

$$
\begin{array}{r}
\boldsymbol{\Pi}_{0}^{(\infty)} \mathbf{B}+\boldsymbol{\Pi}_{1}^{(\infty)} \mathbf{A}_{2}=\mathbf{0} \\
\boldsymbol{\Pi}_{n-1}^{(\infty)} \mathbf{A}_{0}+\boldsymbol{\Pi}_{n}^{(\infty)} \mathbf{A}_{1}+\boldsymbol{\Pi}_{n+1}^{(\infty)} \mathbf{A}_{2}=\mathbf{0}, n \geq 1 .
\end{array}
$$

Under the stability condition, the steady-state solution exists, and it is given by [26]:

$$
\boldsymbol{\Pi}_{n}^{(\infty)}=\boldsymbol{\Pi}_{0}^{(\infty)} \mathbf{R}^{n}, n \geq 1,
$$

where $\mathbf{R}$ is a square matrix rate of order $M_{R T}+1$. Since the spectral radius of $\mathbf{R}$ is strictly lower than 1 , the vector $\boldsymbol{\Pi}_{0}^{(\infty)}$ is obtained by the boundary equation (22) and the normalization condition (20). The matrix $\mathbf{R}$ is a minimal nonnegative solution of the equation

$$
\mathbf{A}_{0}+\mathbf{R} \mathbf{A}_{1}+\mathbf{R}^{2} \mathbf{A}_{2}=\mathbf{0} .
$$

In order to obtain the eigenvalues of $\mathbf{R}$, we define $\mathbf{T}(z)(\forall z \in \mathbf{R})$ as a quadratic tri-diagonal polynomial matrix of the following form:

$$
\mathbf{T}(z)=\mathbf{A}_{0}+z \mathbf{A}_{1}+z^{2} \mathbf{A}_{2}
$$

\section{The case for finite number of BE calls}

Since $\mathbf{Q}^{(f)}$ is aperiodic and irreducible generator, it has a unique stationary probability distribution defined as a solution of the equations

$$
\begin{aligned}
\boldsymbol{\Pi}^{(f)} \mathbf{Q}^{(f)} & =\mathbf{0}, \\
\boldsymbol{\Pi}^{(f)} \mathbf{e}^{(f)} & =1,
\end{aligned}
$$

where $\mathbf{e}^{(f)}$ is a column vector of the ones with an appropriate dimension and $\boldsymbol{\Pi}^{(f)}=\left(\boldsymbol{\Pi}_{0}^{(f)}, \ldots\right.$, $\left.\boldsymbol{\Pi}_{M_{B E}}^{(f)}\right)$. Thus, we obtain the following system:

$$
\begin{array}{r}
\boldsymbol{\Pi}_{0}^{(f)} \mathbf{B}+\boldsymbol{\Pi}_{1}^{(f)} \mathbf{A}_{2}=\mathbf{0} \\
\boldsymbol{\Pi}_{n-1}^{(f)} \mathbf{A}_{0}+\boldsymbol{\Pi}_{n}^{(f)} \mathbf{A}_{1}+\boldsymbol{\Pi}_{n+1}^{(f)} \mathbf{A}_{2}=\mathbf{0} \\
\boldsymbol{\Pi}_{M_{B E}-1}^{(f)} \mathbf{A}_{0}+\boldsymbol{\Pi}_{M_{B E}}^{(f)} \mathbf{F}=\mathbf{0}
\end{array}
$$

where $n=1, \ldots, M_{B E}-1$.

If $\lambda_{B E} \neq \sum_{i=0}^{M_{R T}} v(i) q_{i}$, then the steady-state probability vector $\Pi_{j}^{(f)}$ of BE calls can be written as follows:

$$
\boldsymbol{\Pi}_{j}^{(f)}=\overline{\mathbf{x}} \mathbf{R}^{j}+\overline{\mathbf{y}} \mathbf{S}^{M_{B E}-j}, 0 \leq j \leq M_{B E},
$$

where $\overline{\mathbf{x}}$ and $\overline{\mathbf{y}}$ are the vectors of order $M_{R T}+1$ and the matrices $\mathbf{R}$ and $\mathbf{S}$ are the minimal nonnegative solutions, respectively, to the following equations:

$$
\begin{aligned}
\mathbf{A}_{0}+\mathbf{R} \mathbf{A}_{1}+\mathbf{R}^{2} \mathbf{A}_{2} & =\mathbf{0} \\
\mathbf{S}^{2} \mathbf{A}_{0}+\mathbf{S} \mathbf{A}_{1}+\mathbf{A}_{2} & =\mathbf{0}
\end{aligned}
$$

In order to obtain the eigenvalues of $\mathbf{R}$ and $\mathbf{S}$, we define the quadratic matrix polynomials $\mathbf{T}(z)$ and $\mathbf{T}^{\prime}(z)$, for $z \in \mathbf{R}$ as:

$$
\begin{aligned}
\mathbf{T}(z) & =\mathbf{A}_{0}+z \mathbf{A}_{1}+z^{2} \mathbf{A}_{2}, \\
\mathbf{T}^{\prime}(z) & =\mathbf{A}_{2}+z \mathbf{A}_{1}+z^{2} \mathbf{A}_{0} .
\end{aligned}
$$

\subsection{Summary of Results}

In our analysis we use a spectral analysis approach to rewrite the equation (24) for infinite case and the equation (32) for finite case, respectively, to spectral expansion form:

1. Infinite of BE calls

$$
\boldsymbol{\Pi}_{j}^{(\infty)}=\sum_{i=0}^{M_{R T}} \beta_{i} \phi_{i}^{j} \mathbf{r}_{i}, j=0,1,2, \ldots
$$

where $\phi_{0}, \ldots, \phi_{M_{R T}}$ are the eigenvalues corresponding to the left eigenvectors $\mathbf{\Upsilon}_{0}, \ldots, \mathbf{\Upsilon}_{M_{R T}}$ of the matrix $\mathbf{R}$, i.e., $\mathbf{\Upsilon}_{i} \mathbf{R}=\phi_{i} \mathbf{\Upsilon}_{i}$ and $\beta_{0}, \ldots, \beta_{M_{R T}}$ are the real coefficients.

2. Finite of BE calls

$$
\begin{aligned}
\boldsymbol{\Pi}_{j}^{(f)} & =\sum_{i=0}^{M_{R T}} \beta_{i}\left(\phi_{i}\right)^{j} \Upsilon_{i} \\
& +\sum_{i=M_{R T}+1}^{2 M_{R T}+2} \beta_{i}\left(\frac{1}{\phi_{i}}\right)^{M_{B E}-j} \Upsilon_{i} \\
j & =0,1, \ldots, M_{B E}
\end{aligned}
$$

where $1 / \phi_{M_{R T}+1}, \ldots, 1 / \phi_{2 M_{R T}+2}$ are the eigenvalues corresponding to the left eigenvectors $\mathbf{\Upsilon}_{M_{R T}+1}, \ldots, \mathbf{\Upsilon}_{2 M_{R T}+2}$ of the matrix $\mathbf{S}$ and the $\beta_{M_{R T}+1}, \ldots, \beta_{2 M_{R T}+2}$ are the real coefficients. 
In order to find the unknown parameters eigenvalues, eigenvectors and the coefficients $\beta_{i}$ appearing in both equations (37) and (38), we present several Lemmas and Theorem in the Subsection 4.2

\subsection{Spectral Analysis}

In this subsection, we investigate the computation of the eigenvalues of matrices $\mathbf{R}$ and $\mathbf{S}$.

\section{Lemma 1}

1. The non-zero roots of $\operatorname{det}[\mathbf{T}(z)]$ are the same as the roots of $\operatorname{det}\left[\left(\mathbf{T}^{\prime}(1 / z)\right]\right.$.

2. The eigenvalues of $\mathbf{R}$ of order $m_{i}$ are equal to the roots of the polynomial det $[\mathbf{T}(z)]$ with the order greater than or equal to $m_{i}$.

3. If the eigenvector $\Upsilon_{i}$ of the matrix $\mathbf{R}$ corresponding to the eigenvalue $\phi_{i}$, then $\Upsilon_{i}$ is a left null vector of $\mathbf{T}\left(\phi_{i}\right)$.

4. If $\phi_{i} \neq 0$ and $\frac{1}{\phi_{i}}$ is an eigenvalue of $\mathbf{S}$ with algebraic multiplicity $m_{i}$, then $\phi_{i}$ is a root of the polynomial $\operatorname{det}[\mathbf{T}(z)]$ and its multiplicity is at least $m_{i}$. If $\mathbf{\Upsilon}_{i}$ is an eigenvector of matrix $\mathbf{S}$ corresponding to the eigenvalue $\frac{1}{\phi_{i}}$, then $\mathbf{\Upsilon}_{i}$ is a left null vector of $\mathbf{T}\left(\phi_{i}\right)$.

\section{Proof of Lemma 1}

1. Let $z \neq 0$, then $\mathbf{T}(z)=z^{2} \mathbf{T}^{\prime}(1 / z)$. The nonzero roots of $\operatorname{det}[\mathbf{T}(z)]$ are exactly the roots of $\operatorname{det}\left[\mathbf{T}^{\prime}(1 / z)\right]$.

2. From (25), we have $\mathbf{A}_{0}=-\mathbf{R}\left(\mathbf{A}_{1}+\mathbf{R} \mathbf{A}_{2}\right)$. Replacing it in (26), adding and subtracting $z \mathbf{R A}_{2}$, we get

$$
\begin{aligned}
\mathbf{T}(z) & =-\mathbf{R}\left(\mathbf{A}_{1}+\mathbf{R} \mathbf{A}_{2}\right)+z\left(\mathbf{A}_{1}+z \mathbf{A}_{2}\right) \\
& +z \mathbf{R} \mathbf{A}_{2}-z \mathbf{R} \mathbf{A}_{2} .
\end{aligned}
$$

Thus, we have

$$
\mathbf{T}(z)=(z \mathbf{I}-\mathbf{R})\left(\mathbf{A}_{1}+\mathbf{R} \mathbf{A}_{2}+z \mathbf{A}_{2}\right) .
$$

We calculate the determinant of matrix $\mathbf{T}(z)$ as follows:

$$
\operatorname{det}[\mathbf{T}(z)]=\operatorname{det}(z \mathbf{I}-\mathbf{R}) \operatorname{det}\left(\mathbf{A}_{1}+\mathbf{R} \mathbf{A}_{2}+z \mathbf{A}_{2}\right) .
$$

Let $P_{\mathbf{R}}(z)=\operatorname{det}(z \mathbf{I}-\mathbf{R})$ is the polynomial characteristic of the matrix $\mathbf{R}$ and let $\phi_{i}$ be an eigenvalue of $\mathbf{R}$ of order $m_{i}$. At $z=\phi_{i}$ we have:

$$
\operatorname{det}\left[\mathbf{T}\left(\phi_{i}\right)\right]=P_{\mathbf{R}}\left(\phi_{i}\right) \operatorname{det}\left(\mathbf{A}_{1}+\mathbf{R} \mathbf{A}_{2}+\phi_{i} \mathbf{A}_{2}\right) .
$$

If $\phi_{i}$ is the root of $\operatorname{det}\left(\mathbf{A}_{1}+\mathbf{R} \mathbf{A}_{2}+z \mathbf{A}_{2}\right)$ of order $n_{i}$, then we can write

$$
\operatorname{det}[\mathbf{T}(z)]=\left(z-\phi_{i}\right)^{m_{i}+n_{i}} g(z)
$$

where $g(z)$ is a polynomial of degree $M_{R T}+$ $1-m_{i}-n_{i}$. Therefore, $\operatorname{det}\left[\mathbf{T}\left(\phi_{i}\right)\right]=0$, where $\phi_{i}$ is a root of order $m_{i}+n_{i}$.

3. Let $\Upsilon_{i}$ be the eigenvector corresponding to the eigenvalue $\phi_{i}$. Multiplying (26) by $\mathbf{\Upsilon}_{i}$, using $\mathbf{\Upsilon}_{i} \mathbf{R}=\phi_{i} \mathbf{\Upsilon}_{i}$ and (33), we have

$$
\begin{aligned}
\Upsilon_{i} \mathbf{T}\left(\phi_{i}\right) & =\mathbf{\Upsilon}_{i} \mathbf{A}_{0}+\phi_{i} \mathbf{\Upsilon}_{i} \mathbf{A}_{1}+\phi_{i}^{2} \mathbf{\Upsilon}_{i} \mathbf{A}_{2} \\
& =\Upsilon_{i} \mathbf{A}_{0}+\mathbf{\Upsilon}_{i} \mathbf{R} \mathbf{A}_{1}+\Upsilon_{i} \mathbf{R}^{2} \mathbf{A}_{2} \\
& =\mathbf{\Upsilon}_{i}\left(\mathbf{A}_{0}+\mathbf{R} \mathbf{A}_{1}+\mathbf{R}^{2} \mathbf{A}_{2}\right) \\
& =\mathbf{0}
\end{aligned}
$$

which completes the proof.

4. We can see that $\mathbf{T}^{\prime}(z)=(z \mathbf{I}-\mathbf{S})((z \mathbf{I}+$ $\left.\mathbf{S}) \mathbf{A}_{0}+\mathbf{A}_{1}\right)$. Let $\frac{1}{\phi_{i}}$ be an eigenvalue of $\mathbf{S}$, then $\frac{1}{\phi_{i}}$ is a root of $\operatorname{det}\left[\mathbf{T}^{\prime}(z)\right]$ and its multiplicity at least $m_{i}$. On the other hand, we have

$$
\begin{aligned}
\mathbf{\Upsilon}_{i} \mathbf{T}\left(\phi_{i}\right) & =\Upsilon_{i} \phi_{i}^{2} \mathbf{T}^{\prime}\left(1 / \phi_{i}\right) \\
& =\phi_{i}^{2}\left(\frac{1}{\phi_{i}} \Upsilon_{i}-\Upsilon_{i} S\right)\left(\left(\frac{1}{\phi_{i}} \mathbf{I}+\mathbf{S}\right) \mathbf{A}_{0}+\mathbf{A}_{1}\right) \\
& =\mathbf{0} .
\end{aligned}
$$

The last equality follows from $\Upsilon_{i} \mathbf{S}=\frac{1}{\phi_{i}} \Upsilon_{i}$, which completes the proof.

\section{Lemma 2}

1. At $z=0$, the matrix $\mathbf{T}(z)$ has one eigenvalue $\lambda_{B E}$ of multiplicity $M_{R T}+1$.

2. If $z \neq 0$, the matrix $\mathbf{T}(z)$ has $M_{R T}+1$ different eigenvalues.

3. The determinant of the matrix $\mathbf{T}(z)$ can be written as:

$$
\operatorname{det}[\mathbf{T}(z)]=(1-z) d(z),
$$

where $d(z)$ is a polynomial of degree $2 M_{R T}+$ 1. 


\section{Proof of Lemma 2}

1. From the definition of the polynomial $\mathbf{T}(z)$, we have $\mathbf{T}(0)=\mathbf{A}_{0}$ and if $\lambda$ is real, then the polynomial characteristic of $\mathbf{T}(0)$ becomes

Consequently $\lambda=\lambda_{B E}$ is an eigenvalue of order $M_{R T}+1$ of $\mathbf{T}(z)$ at $z=0$.

2. For $z \in \mathbf{R}^{*}=\mathbf{R}-\{0\}$, we seek $\mathbf{D}=$ $\operatorname{diag}\left(d_{i}\right)$ a diagonal matrix such that the matrix $\mathbf{D T}(z) \mathbf{D}^{-1}$ becomes symmetric. To find the matrix $\mathbf{D}$, one just needs to calculate the diagonal elements, which are obtained as follows: $\frac{d_{i}}{d_{0}}=\sqrt{\left(\frac{\lambda_{R T}}{\mu_{R T}}\right)^{i} \frac{1}{i !}}$, for $i=0, \ldots, M_{R T}$. Let $\mathbf{E}(z)=\mathbf{D T}(z) \mathbf{D}^{-1}$, hence the matrix $\mathbf{E}(z)$ is a tri-diagonal matrix and its elements are given by

$$
\begin{aligned}
\mathbf{E}(z)[i, i]= & k(i, z), \text { for } i=0, \ldots, M_{R T}, \\
\mathbf{E}(z)[i, i+1]= & z \sqrt{i \lambda_{R T} \mu_{R T}}, \\
& i=0, \ldots, M_{R T}-1, \\
\mathbf{E}(z)[i, i-1]= & z \sqrt{(i-1) \lambda_{R T} \mu_{R T}}, \\
& i=1, \ldots, M_{R T},
\end{aligned}
$$

where

$$
\begin{aligned}
k(i, z)= & \lambda_{B E}-z\left(\lambda_{R T}+\lambda_{B E}+i \mu_{R T}\right. \\
& +v(i))+z^{2} v(i), \\
& i=0,1, \ldots, M_{R T}-1, \\
k\left(M_{R T}, z\right)= & \lambda_{B E}-z\left(\lambda_{B E}+M_{R T} \mu_{R T}\right. \\
& \left.+v\left(M_{R T}\right)\right)+z^{2} v\left(M_{R T}\right) .
\end{aligned}
$$

$P_{\mathbf{A}_{0}}(\lambda)=\operatorname{det}\left(\lambda \mathbf{I}-\mathbf{A}_{0}\right)=\left(\lambda-\lambda_{B E}\right)^{M_{R T}+1}$.

Since the matrix $\mathbf{E}(z)$ is real and symmetric of order $M_{R T}+1$, then $\mathbf{E}(z)$ has $M_{R T}+1$ eigenvectors associated with $M_{R T}+1$ real eigenvalues (as described in [6]). However, these eigenvectors form an orthonormal unit. Therefore they constitute a free family. Consequently, $\mathbf{E}(z)$ has $M_{R T}+1$ different eigenvalues.

3. The determinant of matrix $\mathbf{T}(z)$ can be rewritten as given by (46) below. Now, replacing the last column of det $[\mathbf{T}(z)]$ by the sum of all other columns and after simplification, we obtain:

$$
\operatorname{det}[\mathbf{T}(z)]=(1-z) d(z),
$$

where $d(z)$ is given in (48) below which completes the proof.

Since the matrices $\mathbf{E}(z)$ and $\mathbf{T}(z)$ are similar, they have the same eigenvalues which are, say, $\theta_{0}(z), \ldots, \theta_{M_{R T}}(z)$. Let $P_{\mathbf{T}(z)}(\lambda)=\operatorname{det}(\lambda \mathbf{I}-$ $\mathbf{T}(z))$ be the polynomial characteristic of the polynomial matrix $\mathbf{T}(z)$ with a real $\lambda$. Let $\operatorname{Tr}(\mathbf{A})$ denote the trace of the matrix A. According to Lemma 4.2, we get

$$
P_{\mathbf{T}(z)}(\lambda)=\left(\lambda-\theta_{0}(z)\right)\left(\lambda-\theta_{1}(z)\right) \cdots\left(\lambda-\theta_{M_{R T}}(z)\right) \text {. }
$$

and the trace of $\mathbf{T}(z)$ is given by

$$
\begin{aligned}
\operatorname{Tr}[\mathbf{T}(z)] & =\left(M_{R T}+1\right) \lambda_{B E}-M_{R T} \lambda_{R T} \\
& \times \sum_{i=0}^{M_{R T}}\left(\lambda_{B E}+i \mu_{R T}+v(i)\right) z+\sum_{i=0}^{M_{R T}} v(i) z^{2} .
\end{aligned}
$$

$$
\operatorname{det}[\mathbf{T}(z)]=\left|\begin{array}{ccccc}
k(0, z) & z \lambda_{R T} & 0 & \ldots & 0 \\
z \mu_{R T} & k(1, z) & z \lambda_{R T} & \ddots & \vdots \\
0 & \ddots & \ddots & \ddots & 0 \\
\vdots & \ddots & \ddots & \ddots & z \lambda_{R T} \\
0 & \ldots & 0 & z M_{R T} \mu_{R T} & k\left(M_{R T}, z\right)
\end{array}\right|
$$

$$
d(z)=\left|\begin{array}{cccccc}
k(0, z) & z \lambda_{R T} & 0 & \ldots & 0 & \left(\lambda_{B E}-z v(0)\right) \\
z \mu_{R T} & k(1, z) & z \lambda_{R T} & \ddots & \vdots & \left(\lambda_{B E}-z v(1)\right) \\
0 & \ddots & \ddots & \ddots & 0 & \vdots \\
\vdots & \ddots & \ddots & & z \lambda_{R T} & \vdots \\
\vdots & \vdots & 0 & z\left(M_{R T}-1\right) \mu_{R T} & k\left(M_{R T}-1, z\right) & \left(\lambda_{B E}-z v\left(M_{R T}-1\right)\right) \\
0 & 0 & \ldots & 0 & z M_{R T} \mu_{R T} & \left(\lambda_{B E}-z v\left(M_{R T}\right)\right)
\end{array}\right|
$$


Thus, we have

$$
\begin{aligned}
P_{\mathbf{T}(z)}(\lambda)= & \alpha_{0}(z)+\alpha_{1}(z) \lambda+\cdots+ \\
& \alpha_{M_{R T}-1}(z) \lambda^{M_{R T}-1}+\lambda^{M_{R T}},
\end{aligned}
$$

where $\alpha_{i}(z)$ is a continuous polynomial of degree 2, since the trace is $\operatorname{Tr}[\mathbf{T}(z)]=\alpha_{M_{R T}-1}(z)$ (see[6]) and the function $\alpha_{M_{R T}-1}(z)$ is also continuous. Since $\alpha_{M_{R T}-1}(z)=\sum_{i=0}^{M_{R T}} \theta_{i}(z)$, we deduce the continuity of the eigenvalues $\theta_{i}(z)$ $(\forall z \in \mathbf{R})$. We note that at $\lambda=0, P_{\mathbf{T}(z)}(0)=$ $\operatorname{det}[\mathbf{T}(z)]=\alpha_{0}(z)$ and we have

$$
\operatorname{det}[\mathbf{T}(z)]=\theta_{0}(z) \theta_{1}(z) \cdots \theta_{M_{R T}}(z) .
$$

It can be shown that the $\theta_{k}(z)$ are continuous functions of $z$. Furthermore, we can write the eigenvalues according to the following orders (see Lemma 2):

1. If $z \neq 0$, then we have

$$
\theta_{0}(z)<\theta_{1}(z)<\cdots<\theta_{M_{R T}}(z) .
$$

2. If $z=0$, then we have

$$
\theta_{0}(0)=\theta_{1}(0)=\cdots=\theta_{M_{R T}}(0)=\lambda_{B E} .
$$

\section{Lemma 3}

1. All the eigenvalues $\theta_{i}(z)$ are negative at $z=1$, i.e., $\theta_{i}(1) \leq 0$, for $i=0,1, \ldots, M_{R T}$.

2. For $z \in] 0,1\left[\right.$, the eigenvalues $\theta_{i}(z)=0$ have at least one solution, i.e., $\left.\exists \psi_{i} \in\right] 0,1[$ so that $\theta_{i}\left(\psi_{i}\right)=0$, for $i=0,1, \ldots, M_{R T}$.

\section{Proof of Lemma 3}

1. Note that $\mathbf{Q}=\mathbf{T}(1)$, where $\mathbf{Q}=\left(a_{i, j}\right)$, $0 \leq i, j \leq M_{R T}$. Thus, it is seen that diagonal elements of the matrix $\mathbf{Q}$ are all negative. According to the Gersgorin's theorem (see Burden et al. [6]), each eigenvalue $\theta_{i}(1)$ of $\mathbf{T}(1)$ belongs to at least disks whose centers are

$$
a_{i, i}=-\left(\lambda_{R T}+i \mu_{R T}\right)
$$

and the radii are

$$
r_{i}=\lambda_{R T}+i \mu_{R T}, i=0, \ldots, M_{R T} .
$$

Thus, we have $\left|\theta_{i}(1)-a_{i, i}\right| \leq r_{i}$, i.e., $\theta_{i}(1) \leq$ $r_{i}+a_{i, i}=0($ using (53) and (54)) which completes this proof.
2. Combining $\theta_{i}(0)>0, \theta_{i}(1)<0$ and the continuity of these functions, there exists at least one solution $\psi_{i}$ in ]0,1[ such that $\theta_{i}\left(\psi_{i}\right)=0$, which complete the proof.

Theorem 1 The polynomial det $[\mathbf{T}(z)]$ has maximum $2 M_{R T}+2$ roots which are all non-zero. For their localizations, three different cases can be distinguished:

1. If $\lambda_{B E}<\sum_{i=0}^{M_{R T}} v(i) q_{i}$, then only one root at $z=1, M_{R T}+1$ roots are inside the unit disk and $M_{R T}$ outside.

2. If $\lambda_{B E}=\sum_{i=0}^{M_{R T}} v(i) q_{i}$, there is a root of order 2 located at $z=1, M_{R T}+1$ roots are inside the unit disk and $M_{R T}-1$ outside.

3. If $\lambda_{B E}>\sum_{i=0}^{M_{R T}} v(i) q_{i}, M_{R T}$ roots are inside the unit disk, one at $z=1$ and $M_{R T}+1$ outside.

\section{Proof of Theorem 1}

1. If $\lambda_{B E}<\sum_{i=0}^{M_{R T}} v(i) q_{i}$, then the spectral radius of $\mathbf{R}$ is strictly less than one $(\operatorname{sp}(\mathbf{R})<1)$ as shown by Neuts [26, Theorem 3.1.1]. From the Lemma 2, all the eigenvalues of $\mathbf{R}$ are the roots of the $\operatorname{det}[\mathbf{T}(z)]$. Then, there are $M_{R T}+1$ roots of the polynomial $\operatorname{det}[\mathbf{T}(z)]$ in the unit disk for $|z|<1$. These eigenvalues are all real and strictly positive. There is one root at $z=1$ of $\operatorname{det}[\mathbf{T}(z)]$ according to Lemmas 2. The eigenvalues of $\mathbf{S}$ are all the roots of $\operatorname{det}[\mathbf{T}(z)]$, for $|z|>1$.

2. From Lemma 2, we have

$$
\operatorname{det}[\mathbf{T}(z)]=(1-z) d(z),
$$

where $d(z)$ is a polynomial of degree $2 M_{R T}+$ 1. From (48), at $z=1$, we deduce

$$
\begin{aligned}
d(1)= & (-1)^{M_{R T}+1}\left(\mu_{R T}\right)^{M_{R T}} \times \\
& \sum_{i=0}^{M_{R T}}\left(\lambda_{B E}-v(i)\right)\left(\frac{\lambda_{R T}}{\mu_{R T}}\right)^{i} \frac{M_{R T} !}{i !} .
\end{aligned}
$$

By replacing $\left(\frac{\lambda_{R T}}{\mu_{R T}}\right)^{i}$ by $\frac{q_{i}}{q_{0}} i$ ! in the above 
equation, we get

$$
\begin{aligned}
d(1) & =(-1)^{M_{R T}+1}\left(\mu_{R T}\right)^{M_{R T}} \frac{M_{R T} !}{q_{0}} \\
& \times \sum_{i=0}^{M_{R T}}\left(\lambda_{B E} q_{i}-v(i) q_{i}\right), \\
& =(-1)^{M_{R T}+1}\left(\lambda_{B E}-\omega\right) \frac{M_{R T} !}{q_{0}}\left(\mu_{R T}\right)^{M_{R T}},
\end{aligned}
$$

where $\omega=\sum_{i=0}^{M_{R T}} v(i) q_{i}$. If $\lambda_{B E}=\omega$, from (56), we have $d(1)=0$. So there is a root of order 2 at $z=1, M_{R T}+1$ roots in the unit disk and the remaining roots $M_{R T}-1$ are outside the disk.

3. By the same argument to 1 , we can prove the third case.

\section{Calculation of $\beta_{i}$ coefficients}

The above theorem enables us to identify the eigenvalues and the corresponding eigenvectors of the matrices $\mathbf{R}$ and $\mathbf{S}$. Then, these eigenvalues and eigenvectors become known in both equations (37) and (38). But, it remains to find the coefficients $\beta_{i}$ defined in these equations.

\section{The case for infinite number of BE calls}

The matrix $\mathbf{R}$ has $M_{R T}+1$ simple eigenvalues $\phi_{0}, \ldots, \phi_{M_{R T}}$. According to Jordan decomposition, there exists a matrix $\mathbf{V}$ of eigenvectors of $\mathbf{R}$ which is invertible such that

$$
\mathbf{R}=\mathbf{V}^{-1} \boldsymbol{\Phi} \mathbf{V},
$$

where

$$
\begin{aligned}
\boldsymbol{\Phi} & =\operatorname{diag}\left(\phi_{0}, \ldots, \phi_{M_{R T}}\right), \\
\mathbf{V} & =\left(\begin{array}{c}
\mathbf{\Upsilon}_{0} \\
\vdots \\
\mathbf{\Upsilon}_{M_{R T}}
\end{array}\right) .
\end{aligned}
$$

Then (37) becomes

$$
\boldsymbol{\Pi}_{j}^{(\infty)}=\boldsymbol{\Pi}_{0}^{(\infty)} \mathbf{V}^{-1} \boldsymbol{\Phi}^{j} \mathbf{V} .
$$

Hence, we can write the equation (37) in the matrix form:

$$
\boldsymbol{\Pi}_{j}^{(\infty)}=\overline{\boldsymbol{\beta}} \boldsymbol{\Phi}^{j} \mathbf{V}, j=0,1, \ldots,
$$

where

$$
\overline{\boldsymbol{\beta}}=\left(\beta_{0}, \beta_{1}, \ldots, \beta_{M_{R T}}\right)=\mathbf{\Pi}_{0}^{(\infty)} \mathbf{V}^{-1} .
$$

Furthermore, the normalization condition becomes

$$
\sum_{j=0}^{\infty} \pi_{j}^{(\infty)} \overline{1}=\sum_{i=0}^{M_{R T}} \frac{\beta_{i}}{1-\phi_{i}} \Upsilon_{i} \overline{1}=1,
$$

where $\overline{1}$ is a column vector of ones of order $M_{R T}+1$. By combining the expression (58) with the boundary equation (22) and the normalization condition (59), the coefficients $\beta_{k}$ are solution of the linear system

$$
\overline{\boldsymbol{\beta}}\left[\mathbf{V B}+\boldsymbol{\Phi V A _ { 2 }}, \boldsymbol{\Psi V} \overline{1}\right]=[\mathbf{0}, 1],
$$

where

$$
\boldsymbol{\Psi}=\operatorname{diag}\left(\frac{1}{1-\phi_{i}} ; i=0,1, \ldots, M_{R T}\right) .
$$

\section{The case for finite number of BE calls}

By using the Jordan decomposition, we have:

$$
\mathbf{S}=\mathbf{W}^{-1} \mathbf{\Omega W},
$$

where

$$
\begin{aligned}
\mathbf{\Omega} & =\operatorname{diag}\left(\frac{1}{\phi_{i}} ; i=M_{R T}+1, \ldots, 2 M_{R T}+2\right), \\
\mathbf{W} & =\left(\begin{array}{c}
\Upsilon_{M_{R T}+1} \\
\vdots \\
\mathbf{\Upsilon}_{2 M_{R T}+2}
\end{array}\right) .
\end{aligned}
$$

Since $\lambda_{B E} \neq \sum_{i=0}^{M_{R T}} v(i) q_{i}$ and the $M_{R T}+1$ eigenvalues of matrix $\mathbf{R}$ are singles and nonzeros, then (32) for $j=0,1, \ldots, M_{B E}$ can be rewritten

$$
\boldsymbol{\Pi}_{j}^{(f)}=\overline{\mathbf{x}} \mathbf{V}^{-1} \boldsymbol{\Phi}^{j} \mathbf{V}+\overline{\mathbf{y}} \mathbf{W}^{-1} \boldsymbol{\Omega}^{M_{B E}-j} \mathbf{W} .
$$

Now, we deduce the form close of the steadystate probability vector in finite case as

$$
\boldsymbol{\Pi}_{j}^{(f)}=\sum_{i=0}^{M_{R T}} \beta_{i} \phi_{i}^{j} \Upsilon_{i}+\sum_{i=M_{R T}+1}^{2 M_{R T}+2} \frac{\beta_{i}}{\phi_{i}^{M_{B E}-j}} \Upsilon_{i}
$$

By combining the expression from (61) with the boundary conditions (29), (31) and the normalized condition (28), we drive a set of equations which uniquely determines the coefficients $\beta_{0}, \ldots, \beta_{2 M_{R T}+2}$. 


\section{Multi-cell Case}

In this section, we extend the previous analysis to the multi-cell case by including an approximation for the other cell interference $\left(I_{\text {other }}\right)$. We consider here a multi-cells network composed by $K+1$ cells. Assume that all cells are identical and homogeneous. Since the number of class in a cell is random and depends on the arrivals of calls and exponential call duration, it is not reasonable to assume the relation (3) is valid at each moment. Instead, we assume that it holds in expectation, i.e., $E\left[I_{\text {other }}\right]=g E\left[I_{\text {own }}\right]$. This assumption is especially reasonable in high density of mobiles. However the minimal received power $\left(P_{i}\right)$ given by equation (4) becomes

$$
P_{i}=\frac{N \Delta_{i}}{1-X_{R T} \Delta_{R T}-X_{B E} \Delta_{B E}-g E\left[I_{o w n}\right]}
$$

where

$$
\begin{aligned}
E\left[I_{\text {own }}\right] & =E\left[X_{R T} \Delta_{R T}\right]+E\left[X_{B E} \Delta_{B E}\right] \\
& =\sum_{i=0}^{M_{R T}} i q_{i} \Delta_{R T}+\left(1-\boldsymbol{\Pi}_{0}^{B E} \mathbf{e}\right) \\
& \times \sum_{i=0}^{M_{R T}} q_{i} C(i)
\end{aligned}
$$

where $q_{i}$ is given in (11), $\Pi_{0}^{B E}$ is obtained from (58) for infinite case and from (61) for finite case, and $\mathbf{e}$ is a column vector of the ones with an appropriate dimension. The second term in equation (63) follows from the fact that the $\mathrm{BE}$ calls, use all the remaining capacity if there is at least one BE call. The new capacity becomes $\Theta=1-g E\left[I_{\text {own }}\right]$ which is unknown and depends on the steady-state distribution of the system. In order to obtain the total capacity of a cell, we use the following algorithm.

\section{Performance Metrics}

In this section, we present the performance of interest as a function of the steady-state probabilities obtained in the previous sections.

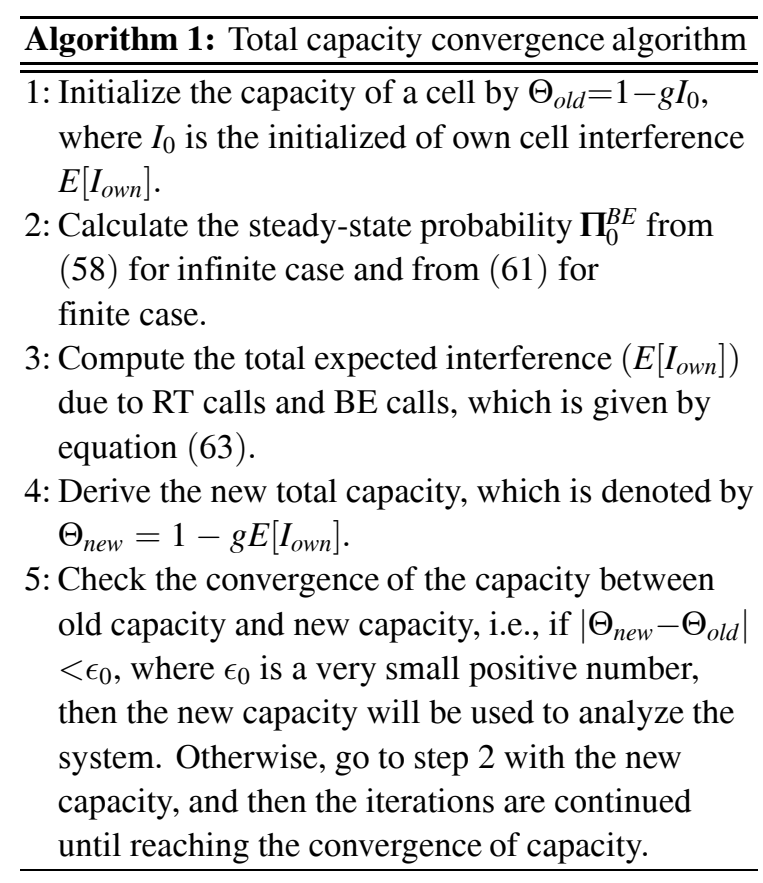

\section{The Case for Infinite Number of BE Calls}

The marginal probability of $\Pi_{j}^{(\infty)}$ is denoted by $P_{j}^{(\infty)}$ as

$$
P_{j}^{(\infty)}=\sum_{i=0}^{M_{R T}} \beta_{i} \phi_{i}^{j} \mathbf{\Upsilon}_{i} \overline{1}
$$

Furthermore, the marginal average number of $\mathrm{BE}$ calls in the system is given by:

$$
E_{B E}^{(\infty)}=\sum_{i=0}^{M_{R T}} \beta_{i} \frac{\phi_{i}}{\left(1-\phi_{i}\right)^{2}} \mathbf{\Upsilon}_{i} \overline{1} .
$$

Using the Little's result, the mean sojourn time of the BE calls is given by

$$
T_{B E}^{(\infty)}=\frac{E_{B E}^{(\infty)}}{\lambda_{B E}} .
$$

\section{The Case for Finite Number of BE Calls}

The marginal probability in this case is

$$
P_{j}^{(f)}=\sum_{i=0}^{M_{R T}} \beta_{i} \phi_{i}^{j} \mathbf{r}_{i} \overline{1}+\sum_{i=M_{R T}+1}^{2 M_{R T}+2} \frac{\beta_{i}}{\phi_{i}^{M_{B E}-j}} \mathbf{r}_{i} \overline{1}
$$

The blocking probability of BE calls can be computed from the above equation considering 
that the number of $\mathrm{BE}$ calls is equal to $M_{B E}$. Therefore, it is given as

$$
P_{M_{B E}}^{(f)}=\sum_{i=0}^{M_{R T}} \beta_{i} \phi_{i}^{M_{B E}} \mathbf{\Upsilon}_{i} \overline{1}+\sum_{i=M_{R T}+1}^{2 M_{R T}+2} \beta_{i} \mathbf{\Upsilon}_{i} \overline{1} .
$$

The marginal average number of BE calls is given by the following formula:

$$
E_{B E}^{(f)}=\sum_{j=0}^{M_{B E}} j P_{j}^{(f)}
$$

Then, by using Little's result, the sojourn time of existing BE calls in the system is obtained as

$$
T_{B E}^{(f)}=\frac{E_{B E}^{(f)}}{\lambda_{B E}\left(1-P_{M_{B E}}^{(f)}\right)} .
$$

\section{Numerical Results and Simulations}

In this section, we present some numerical results and validate the expressions found in the previous sections with a discrete time network simulator (NS2). We consider two classes of calls representing RT calls and BE calls. The first type of calls (i.e., real-time calls) can support adaptive rates. In UMTS, the AMR voice codec that offers eight transmission rates varying between $4.75 \mathrm{Kbps}$ and $12.2 \mathrm{Kbps}$, and that can be dynamically changed every $20 \mathrm{msec}$ (see Holma and Toskala [16]). We work on WCDMA system which is a multiple access system with code distribution. We use direct sequence (DS-WCDMA) modulation where the sequence of bits corresponding to the user data are spread out over a broad band-width by multiplying the data by a pseudo-random sequence of bits (called chips). In order to be able to support very high flows (up to $2 \mathrm{Mbps}$ ) the WCDMA supports transmissions with factor of variable spreading out and multiple codes. The throughput chip (binary rate of the sequence of coding) is taken equal to $3.84 \mathrm{Mcps}$ for a modulation band-width of $5 \mathrm{Mhz}$. The total system capacity is $\Theta_{\epsilon}=1-10^{-5}$ [15]. Demanded normalized rate of loading is given by $R_{T}=\left|\Theta_{\epsilon} / \Delta_{R T}^{m}\right| R_{R T}^{m}$. The BE normalized load is $\bar{\rho}_{B E}=\frac{\lambda_{B E}}{\mu_{B E}} \frac{1}{R_{T}}$, and the RT normalized load is $\bar{\rho}_{R T}=\rho_{R T} \frac{R_{R T}^{m}}{R_{T}}$. In these numerical results, we neglect $\sigma_{\xi}$ in which
$\Gamma$ is equal to 1 . Also, we use in our numerical applications (see Table 1) based on standard WCDMA parameter values[16, 15].

\begin{tabular}{|l||ll|}
\hline$E_{R T} / N_{0}$ & $4.1 \mathrm{~dB}$ \\
\hline$E_{B E} / N_{0}$ & $3.1 \mathrm{~dB}$ \\
\hline$R_{R T}^{m}$ & $4.27 \quad \mathrm{Kbps}$ \\
\hline$R_{R T}^{r}$ & $12.2 \mathrm{Kbps}$ \\
\hline$R_{T}$ & $38 \mathrm{Kbps}$ \\
\hline$W$ & $3.84 \quad \mathrm{Mcps}$ \\
\hline$\lambda_{B E}$ & $10 \quad$ call $/ \mathrm{sec}$ \\
\hline $1 / \mu_{B E}$ & $1 / 5 \quad \mathrm{Mbits}$ \\
\hline $\bar{\rho}_{B E}$ & 0.5 & \\
\hline$\lambda_{R T}$ & $0.3 \quad \mathrm{call} / \mathrm{sec}$ \\
\hline $1 / \mu_{R T}$ & $10 \quad \mathrm{sec}$ \\
\hline $\bar{\rho}_{R T}$ & 0.3 & \\
\hline
\end{tabular}

Table 1. Numerical values

Figure 1 shows the blocking probability of RT calls in terms of the BE threshold reserved capacity $\left(L_{B E}\right)$ for each RT normalized load $\bar{\rho}_{R T}$ in logarithm scale. The results are obtained in special case of mean service time which is fixed at $\mu_{R T}^{-1}=100 \mathrm{sec}$. As expected, when the normalized load of RT calls increases, the blocking probability increases even in small value of $L_{B E}$. According to Figure 1, when $L_{B E}$ increases, the blocking probability of RT calls increases the remaining bandwidth for the RT calls decreases. Furthermore, the probability of blocking increases drastically for different RT normalized loads. This phenomenon is due to the fact that very low resources are remained for RT calls.

We keep the normalized load as constant and vary the holding time of the RT calls $1 / \mu_{R T}$ with $\{100,10,1,0.01\}$ in second unit. We evaluate the performance metrics of interest as a function of BE threshold reserved capacity. Thus, Figure 2 shows in terms of BE threshold reserved capacity for different service rates of RT calls that for small values of $L_{B E}$ a great variation in sojourn time of BE calls. This observation implies that the BE sojourn time is influenced noticeably by service rate of RT calls. However, the decreasing trend is observed only for sojourn times above $0.005 \mathrm{~ms}$. When the sojourn time 
becomes less than $0.005 \mathrm{~ms}$, no considerable differences in sojourn times are observed.

Call Admission Control for BE calls: In this part, we define an admission control for the BE calls. Initially, the operator does not know how to divide the available bandwidth among RT and $\mathrm{BE}$ calls, but it can impose a quality of service over the sojourn time. Figure 3 presents the BE average sojourn time in terms of the BE threshold reserved capacity $\left(L_{B E}\right)$. For small value of $L_{B E}$, we observe that the expected BE sojourn time varies greatly with increasing $L_{B E}$. Then, the lower reserved portion capacity of BE call implies longer BE expected sojourn time. Note that this remark is confirmed also in[2]. Now, we assume that the operator knows how to divide the bandwidth between the RT and BE calls using the information of channel state. In this case, it imposes a quality of service over the sojourn time. In Figure 4, we fix a value of sojourn time and calculate the maximum BE arrival rate noted by $\lambda_{B E}^{*}$ in which the quality of service is satisfied. For small values of $\mathrm{BE}$ threshold of sojourn time, we see that the maximum $\mathrm{BE}$ arrival rate varies greatly with increasing $\mathrm{BE}$ threshold of sojourn time. As it can be seen, the effect of increasing the BE threshold of sojourn time on the maximum $\mathrm{BE}$ arrival rate is less pronounced when the $\mathrm{BE}$ threshold of sojourn time is greater than $0.1 \mathrm{~ms}$.

In this paragraph, we validate the expressions found in the previous sections with a discrete time network simulator NS2 (UMTS extensions for NS2, see[5]). In Figures 5 and 6, we describe the blocking probability of RT calls and the sojourn time of BE calls versus the capacity reserved for the BE calls $\left(L_{B E}\right)$ obtained by simulation and analytical results. In this simulation we used the following parameters:

1. Mean RT call duration is $180 \mathrm{sec}$;

2. Arrival RT call rate is $0.5 \mathrm{call} / \mathrm{sec}$;

3. Mean BE session size is 1.6 Mbits;

4. Arrival BE call rate is $0.5 \mathrm{call} / \mathrm{sec}$.

In Figure 5, the error between the analytical and simulation results is very small i.e. it is close to zero. Whereas in Figure 6 the error between these results is of order of $10^{-2}$ for a small value of the capacity reserved for the $\mathrm{BE}$ calls and it becomes very small when $L_{B E}$ increases $\left(L_{B E}>0.5\right)$. These results are obtained by increasing the number of the simulations and taking their average values in the final results as plotted in this figure. Then the difference between the simulation and the analytic results becomes very small when the $L_{B E}$ is more than 0.5 . We conclude that analytical results matched closely the simulations results.

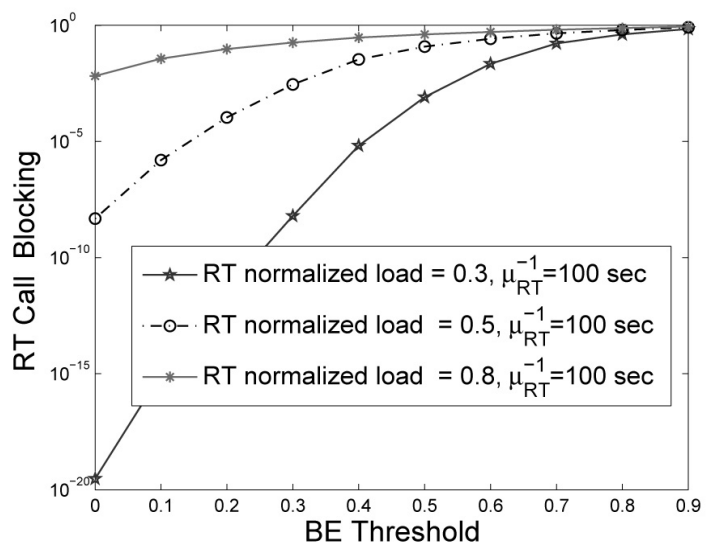

Figure 1. Blocking probability of RT calls in terms of BE threshold reserved capacity $\left(L_{B E}\right)$.

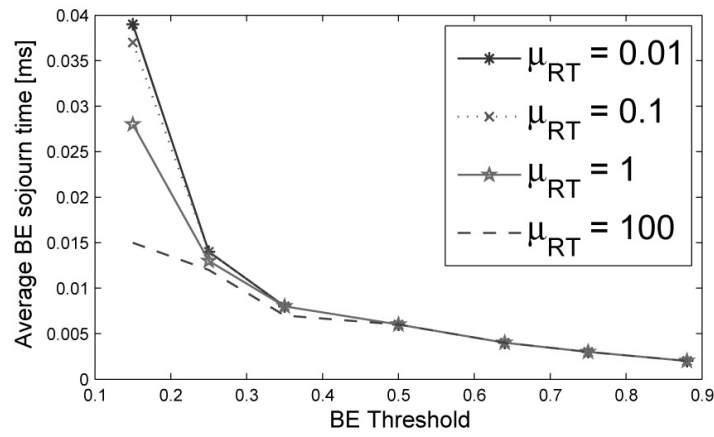

Figure 2. Sojourn time of BE calls in terms of BE threshold reserved capacity $\left(L_{B E}\right)$.

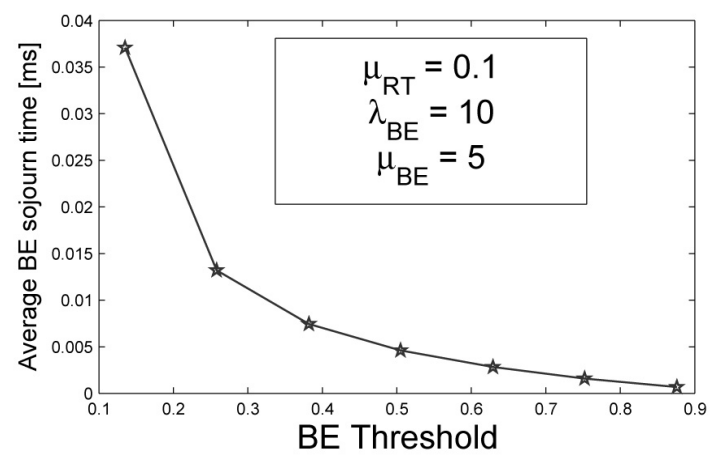

Figure 3. Average sojourn time of the BE calls in terms of capacity reserved for the BE calls $\left(L_{B E}\right)$. 


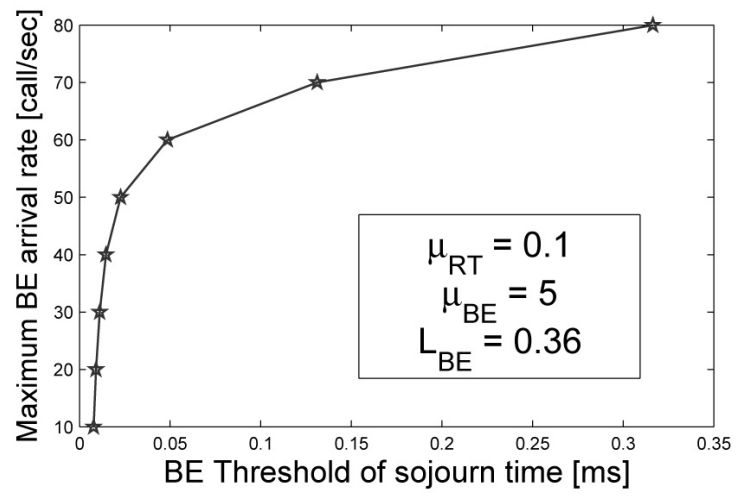

Figure 4. The maximum BE arrival rate versus the threshold of sojourn time.

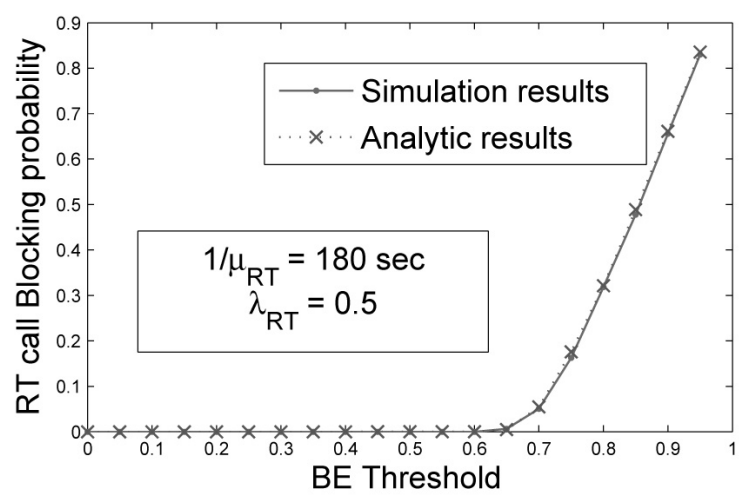

Figure 5. Blocking probability of RT calls in terms of capacity reserved for the BE calls $\left(L_{B E}\right)$, validation of theoretical model: simulation and analytical results.

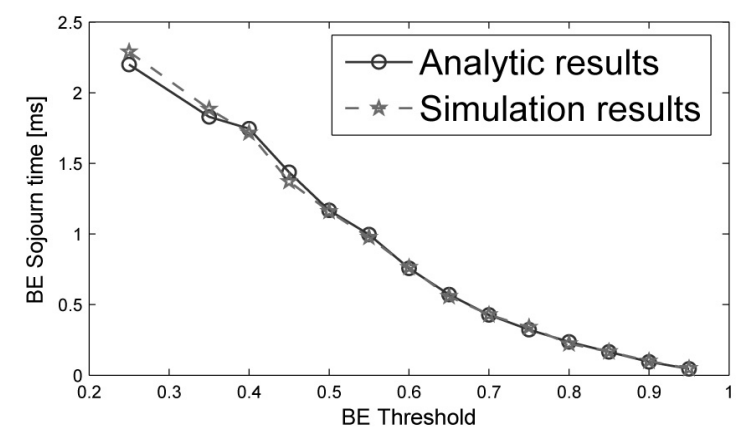

Figure 6. Sojourn time of BE calls in terms of capacity reserved for the BE calls $\left(L_{B E}\right)$, validation of theoretical model: simulation and analytical results.

\section{Conclusions}

In this paper, we carried out an analysis of the capacity evaluation of wireless cellular network, in particular the uplink of WCDMA system by using the two dimensional continuous time
Markov chain (CTMC) technique. We considered two types of calls: real-time and besteffort. Based on spectral analysis approach, we obtain the simultaneous steady-state distribution of the real-time and best-effort calls. This approach provides a closed form expressions for performance metrics: blocking probability, sojourn time and throughput. These metrics are given for both infinite and finite numbers of best-effort calls. This method is efficient and provides an exact solution compared to [15]. Further, we studied the influence of reserved bandwidth of best-effort calls on the system performance. In addition, we evaluated explicitly the probability of existence of best-effort calls for both cases (finite and infinite) as well as the blocking probability of the best-effort calls. Furthermore, we provided some numerical results of system performance and validated these results through simulation by using the network simulator (NS2).

The spectral analysis developed in this paper could be helpful to the design of various aspects of wireless networks. For example, one of these aspects could be the design of Worldwide Interoperability for Microwave Access (WiMAX), where the number of calls can be separated into two classes of services, namely streaming (real-time) calls and elastic (best-effort) calls. Other examples are High Speed Downlink Packet Access (HSDPA) and High Speed Uplink Packet Access (HSUPA), where the calls are usually studied with streaming and elastic calls. We note that the advanced spectral analysis approach is still valid if the wireless network is modeled by a Markov chain of $r$ dimensions with quasi-birth-and-dead (QBD) homogeneous process.

Furthermore, the wireless networks WiMAX and HSPA use the adaptive modulation and coding (AMC) scheme for serving the users with and without mobility. In this case, it will be interesting to analyze the system capacity of these networks with the AMC scheme in the future works. In $[19,28,30]$ we completed a performance evaluation for the IEEE802.16e and HSDPA standards. We focused on the effects of the standardized AMC and code partitioning [29] schemes. Here, we proposed a CAC algorithm where a bandwidth share of the total bandwidth are allocated to ease the migration which consumes more resources. In addition, 
the system seeks to first accept the calls incoming in the inner region, i.e. the calls that require fewer resources. The RT calls are characterized by the same physical bit rate needs, and ask for resource as function of the modulation efficiency of their region. The BE calls share the available bandwidth left by the RT calls and remain in the system in accordance with these resource consumptions. We studied the effect of our proposed CAC algorithm in the AMC and code partitioning environment. The results showed that the existence of a bandwidth share allocated to outer call migrations greatly improves the overall performances. The CAC algorithm largely increases the BE throughput and hence drastically decreases the sojourn time. In addition, we observed that the BE bandwidth allocation have a major impact on the RT blocking probability. However, our study allows the service providers to find a tradeoff as function of their customer needs. In [18], we focused on the UMTS extension HSDPA and presented an approach for serving users with a constant bit rate while changing between different modulation schemes. Our results showed that the reserved bandwidth by Internet Service Provider to manage its network depends largely on the traffic pattern (low blocking and low dropping). These results allowed us to find a tradeoff as function of their customers need. The reserved bandwidth that belongs to this tradeoff gives increase largely to the average throughput and low reject calls.

\section{Acknowledgment}

This work was supported by a research contract with Maroc Telecom R\&D No. 10510005458.06 PI.

\section{References}

[1] G. Alpan, E. Altman, H. Magroun, Kofman, Call Admission Control in the presence of point-tomultipoint best-effort connections. Presented at the Proceedings of ICC, Vancouver, British Columbia, Canada, 1999.

[2] E. Altman, Capacity of multi-service CDMA cellular networks with best-effort applications. Presented at the Proceedings of MOBICOM, Atlanta, Georgia, USA, 2002.
[3] E. Altman, Capacity of multi-service cellular networks with transmission-rate control: a queueing analysis. Presented at the Proceedings of the 8th Annual International Conference on Mobile Computing and Networking, MobiCom, Atlanta, Georgia, USA, 2002.

[4] E. Altman, T. Chahed, S. E. Elayoubi, Joint uplink and downlink capacity considerations in admission control in multiservicee CDMA/HSDPA systems. Presented at the Proceedings of Valuetools, Nante, France, 2007.

[5] P. BAllester, P. Martin, http://www.geocities.com/opahostil/.

[6] R. L. Burden, J. D. FAIRES, Numerical Analysis. Brooks Cole Publishing, San Francisco, ISBN: 10 0534955339, 1998.

[7] T. Chahed, S. E. Elayoubi, E. Altman, On design of TDD for joint uplink and downlink resource allocation in OFDMA-based WiMax. Presented at the Proceedings of Vehicular Technology Conference IEEE 68th VTC, Calgary, Alberta, Canada, 2008.

[8] R. CHAкKA, Spectral expansion solution for some finite capacity queues. Annals of Operations $R e$ search, 79, 27-44, 1998.

[9] J. CHEN, W. JIAO, H. WANG, A service flow management strategy for IEEE 802.16 broadband wireless access systems in TDD mode. Presented at the Proceedings of IEEE International Conference on Communications, ICC, Seoul, Korea, 2005.

[10] L. Ding, J. S. LEHNERT, Calculation of Erlang Capacity for Cellular CDMA Uplink System. Presented at the Proceedings of IEEE Wireless Communications and Networking Conference, WCNC, Chicago, USA, 2000.

[11] E. Elayoubi, T. Chahed, G. G. Hebuterne, Mobility-aware admission control schemes in the downlink of third generation wireless systems. IEEE Transactions on Vehicular Technology, 56, 245259, 2007.

[12] S-E. Elayoubi, T. Chahed, L. Salahdin, Optimization of radio resource management in UMTS using pricing. Computer Communications, 28(15), 1761-1769, 2005.

[13] S-E. Elayoubi, T. Chahed, M. Tlais, A. Samhat, Measurement-based admission control in UMTS. Special Issue of Annals of Telecommunications on Traffic Engineering and Routing, 59(11-12), 14331445, 2004.

[14] R. Elazouzi, E. Altman, A queuing analysis of packet dropping over a wireless link with retransmissions. Presented at the Proceedings of IEEE ICC, Paris, France, 2004.

[15] N. Hegde, E. Altman, Capacity of multiservice WCDMA networks with variable GoS. Wireless Networks, 12, 241-253, 2006.

[16] H. Holma, A. TOSKALA, WCDMA for UMTS. JohnWiley \& Sons, Inc. ISBN: 0470844671, New York, NY, USA, 2002. 
[17] E. S. Hwang, C. H. Cho, H. H. SeO, B. H. RyU, W. N. LEE, A study of code partioning Scheme of efficient random access in OFDMA-CDMA ranging subsystem. In JCCI, p. 262, 2004.

[18] K. Ibrahimi, R. Elazouzi, S. K. Samanta,, E. H. BOUYAKHF, Adaptive Modulation and Coding Scheme with intra- and Inter-cell Mobility in HSDPA Systems. To apear in the IEEE/ICST BROADNETSö09 Sixth Internantional Conference on Broadband Communications, Networks, and Systems, Madrid, Spain, 2009.

[19] K. Ibrahimi, R. El-Azouzi, T. Peyre, E. H. BOUYAKHF, CAC Algorithms Based on Random Waypoint Mobility for IEEE 802.16e Networks. Presented at the Proceedings of IEEE NGNS'09 International Conference on Next Generation Networks and Services, Rabat, Morocco, 2009.

[20] J. LAIHO, A. WACKER, Radio network planning process and methods for WCDMA. Annales des Télécommunications, 56(5-6), 317-331, 2001.

[21] G. LATOUCHE, V. RAMASWAMI, Introduction to matrix analytic methods in stochastic modeling. ISBN 0-89871-425-7 (Paperback), 1999.

[22] N. Mandayam, J. Holtzman, S. BARBeris, Performance and capacity of a voice/data CDMA system with variable bit rate sources. special Issue on Insight into Mobile Multimedia Communications, pages 537-550, Academic Press Inc., San Diego, CA, USA, January 1998.

[23] I. MITRANI, The spectral expansion solution method for Markov processes on lattice strips. In J. H. Dshalalow, editor. Advances in Queueing Theory, Methods and Open Problems, CRC Press, Boca Raton, FL, Chapter 13, pp. 337-352, 1995.

[24] I. Mitrani, R. CHAKKA, Spectral expansion solution for a class of Markov models: Application and comparison with the matrix-geometric method. Performance Evaluation, 23(3), 241-260, 1995.

[25] I. Mitrani, R. CHAKKA, Spectral expansion solution for a finite capacity multiserver system in a markovian environment. Presented at the Proceedings of 3rd International Workshop on Queueing Networks with Finite Capacity, Bradford, UK, 1995.

[26] F. NEUTS, Matrix-geometric solutions in stochastic models. The John Hopkins University Press, Baltimore, Maryland, 1981.

[27] V. PLA, V. CASARES-Giner, Analysis of priority channel assignment schemes in mobile cellular communication systems: a spectral theory approach. Performance Evaluation, 59, 199-224, 2005.

[28] T. Peyre, R. Elazouzi, IEEE 802.16e Cell Capacity Including Mobility Management and QoS Differentiation. Presented at the Proceedings of Wireless Communication and Networking Conference (WCNC), Budapest, Hungary, 2009.

[29] T. Peyre, R. El-AzouzI, T. Chahed, QoS Differentiation for Initial and Bandwidth Request Ranging in 802.16. Presented at the Proceedings of PIMRC, Cannes, France, 2008.
[30] T. Peyre, K. Ibrahimi, R. Elazouzi, IEEE 802.16 Multi-class Capacity Including AMC Scheme and QoS Differentiation for Initial and Bandwidth Request Ranging. Presented at the Proceedings of ICST Conference on Performance Evaluation Methodologies and Tools (ValueTools), Greece, 2008.

[31] R. N. QueIJA, Processing Sharing Models for Integrated-services Networks. PhD Thesis, Eindhoven University of Technology, ISBN: 90-6464667-8, January 2000.

[32] H. H. SEO, B. H. RyU, J. J. Won, C. H. Cho, H. W. LEE, Perfomance analysis of random access protocol in OFDMA-CDMA. Presented at the Proceedings of KICS Fall Conference, Korea, 2003.

[33] A. J. VITERBI, CDMA: Principles of spread spectrum communication. Addison Wesley Longman Publishing Co. Inc., Redwood City, CA, USA, 1995.

[34] H. A. Wang, W. Li, D. P. Agrawal, Dynamic admission control and QoS for 802.16 wireless MAN. Wireless Telecommunications Symposium, pp. 6066, April 2005.

[35] J. YoU, K. KIM, Capacity evaluation of the OFDMA-CDMA ranging subsystem in IEEE 802.16-2004. Presented at the Proceedings of WiMob, Montreal, Canada, 2005.

[36] Q. ZHANG, O. YUE, UMTS air inteface voice/data capacity-part 1: reverse link analysis. Presented at the Proceedings of IEEE Vehicular Technology Conference, Rhodes, Greece, 2001.

Received: November, 2008 Revised: August, 2009 Accepted: September, 2009

Contact addresses:

Abdellatif Kobbane ENSIAS, Mohammed V-Souissi University Avenue Mohammed Ben Abdallah Regragui Madinat Al Irfane, BP 713, Agdal Rabat, Morocco e-mail: kobbane@ensias.ma

Rachid Elazouzi LIA/CERI, University of Avignon 339 Chemin des Meinajaris BP1228 84911 Cedex 9, Avignon, France e-mail: rachid.elazouzi@univ-avignon.fr

Khalil Ibrahimi ${ }^{1,2}$ ${ }^{1}$ LIA/CERI, University of Avignon 339 Chemin des Meinajaris BP1228 84911 Cedex 9, Avignon, France ${ }^{2}$ LIMIARF/FSR, Mohammed V-Agdal University 4 Avenue Ibn Battouta B.P. 1014 Agdal, Rabat, Morocco e-mail: khalil.ibrahimi@univ-avignon.fr

Sujit K. Samanta LIA/CERI, University of Avignon 339 Chemin des Meinajaris BP1228 84911 Cedex 9, Avignon, France e-mail: sujit.samanta@univ-avignon.fr

El-Houssine Bouyakhf LIMIARF/FSR, Mohammed V-Agdal University 4 Avenue Ibn Battouta B.P. 1014 Agdal, Rabat, Morocco e-mail: bouyakhf@fsr.ac.ma 
ABDELLATIF KOBBANE is currently working as assistant professor at the Ecole Nationale Suprieure d'Informatique et d'Analyse des Systemes (Morocco). He received his $\mathrm{PhD}$ degree in computer science from the Mohammed V-Agdal University (Morocco) and the University of Avignon (France) in September 2009. He received his research MS degrees in computer science, Telecommunication and Multimedia from the Mohammed V-Agdal University (Morocco) in 2003. His research interests lie with the field of wireless networking, performance evaluation in wireless network, cognitive radio and quality of services. Dr Kobbane has been on the technical program committee of different IEEE conferences, including ICC, WiMob, WCNC, and local chair of WiMob'09. He was a member of the project framework Maroc Telecom (2006-2009). Dr Kobbane is an IEEE member and President of the Association of Researchers in Information Technology in Rabat Morocco.

RACHID ELAZOUZI received the Ph.D. degree in applied mathematics from the Mohammed V University, Rabat, Morocco (2000). He joined INRIA (National research institute in informatics and control) SophiaAntipolis for post-doctoral and research engineer positions. Since 2003, he has been a researcher at the University of Avignon, France. His research interests are mobile networks, performance evaluation, the TCP protocol, error control in wireless networks, resource allocation, networking games and pricing.

KHALIL IBRAHIMI was born in Arbaoua, Morocco in 1978. He received his B.Sc. degree in mathematical sciences in September, 2003 and his Master Sc. degree in engineering, telecommunications and multimedia (ITM) in December, 2005, all from Faculty of Sciences at Mohammed V University, Agdal, Rabat, Morocco. From October, 2006, he has been working on his Ph.D thesis ("Resource allocation and performance evaluations in next generation wireless networks") in Computer Sciences at University of Avignon and at Mohammed V Agdal university under supervision of Dr. Eitan ALTMAN at the INRIA-Sophia Antipolis, France, Dr. Rachid ELAZOUZI at the laboratory of computer science of Avignon, France and Prof. El-Houssine BOUYAKHF, Rabat Morocco. His research interests include in particular performance evaluation and resources allocation of next generation networks $(3 \mathrm{G}$, Beyond $3 \mathrm{G}$ and $4 \mathrm{G})$, networking game and cognitive radio.

SUJIT KUMAR SAMANTA was born in West Bengal, India, in 1977. He received the B.Sc. and M.Sc. degrees in mathematics from the Vidyasagar University, West Bengal, India, in 1998 and 2000, respectively. Since July 2001, he is working as a Ph.D. student at the Department of Mathematics, Indian Institute of Technology, Kharagpur, India. His main research interests include discrete-time queueing theory and its applications. He has published research articles in Journal of the Operational Research Society, Computers and Mathematics with Applications. He is presently a Postdoctoral Fellow at Laboratory of Computer Science, University of Avignon, France, 2008-2009.

PROF. EL-HOUSSINE BOUYAKHF received the Engineer degree from Sup' Aero (ENSAE) National Higher School of Aeronautics and Space, Toulouse, France in 1976, he also received the Doctor Engineer degree in pattern recognition and artificial intelligence from the University Paul Sabatier, Toulouse, France in 1980 and "Doctorat d'Etat" in robotics and artificial intelligence from LAAS of CNRS and University Paul Sabatier, Toulouse, France in 1988. Since 1988, he works as lecturer at the Faculty of Sciences, Rabat, teaching Computer Sciences, Pattern Recognition, Image Processing and Artificial Intelligence courses. His main topics of interest are: artificial intelligence, pattern recognition, image processing and telecommunications. El Houssine BOUYAKHF is the scientific leader of LIMIARF Lab. He supervises several PhD theses in the research themes listed before. He is coordinator/cocoordinator of Masters in Computer Sciences, Telecommunications and Imaging and in Bioinformatics. 
